\title{
A New Algorithm for the Determinant and the Inverse of Banded Matrices
}

\author{
Mohamed Elouafi ${ }^{1}$, Driss Aiat Hadj Ahmed² \\ ${ }^{1}$ Classes Préparatoites aux Grandes Ecoles d'Ingénieurs, Lycée My Alhassan, Tangier, Morocco \\ ${ }^{2}$ Regional Center for Career Education and Training (CRMEF)-Tangier, Tangier, Morocco \\ Email: med3elouafi@gmail.com, ait hadj@yahoo.com \\ Received **** 2014 \\ Copyright (c) 2014 by authors and OALib. \\ This work is licensed under the Creative Commons Attribution International License (CC BY). \\ http://creativecommons.org/licenses/by/4.0/ \\ (c) (i) Open Access
}

\begin{abstract}
In the current article, the authors present a new recurrence formula for the determinant of a banded matrix. An algorithm for inverting general banded matrices is derived.
\end{abstract}

\section{Keywords}

\section{Banded Matrices, Determinants}

\section{Subject Areas: Mathematical Analysis, Numerical Mathematics}

\section{Introduction}

The aim of this work is to extend the algorithm presented in [1] to obtain the inverse of the banded matrix

$$
\boldsymbol{B}=\left(\begin{array}{ccccccc}
a_{1,1} & \ldots & a_{1, p+1} & 0 & & \ldots & 0 \\
\vdots & \ddots & & a_{2, p+2} & & & \vdots \\
\vdots & & \ddots & & & & \\
a_{q+1,1} & \ddots & & & & \ddots & \\
0 & a_{q+2,2} & \ddots & & & & \\
\vdots & \ddots & \ddots & \ddots & & & \vdots \\
0 & \ddots & & & \ldots & \ldots &
\end{array}\right),
$$

Such matrices arise frequently in many problems such as computing the condition number or the discretization of partial differential equation in $2 D$ or $3 D$ by finite difference [2]. In practice, the bandwidth $p+q$ 


\section{is much less than $n$.}

Standard method to compute the inverse of the matrix $\boldsymbol{B}$ is to use the $L U$ based methods [3] and there are special algorithms taking into account the special form of the matrix [4]. Other methods were proposed more recently [5].

For the $n \times n$ banded matrix $\boldsymbol{B}$, where we assume that $a_{i, p+i} \neq 0$ for $i=1, \cdots, n-p$, we associate the sequence $\left(A_{i, k}\right)_{1 \leq i \leq n+p, 1 \leq k \leq p}$ defined by the following relations for $k=1, \cdots, p$ :

$$
A_{i, k}=\delta_{i, k} \text { for } i=1, \cdots, p \text {, }
$$

and for $i=1, \cdots, n-p$ :

$$
-a_{i, p+i} A_{p+i, k}=\sum_{s=1}^{p+i-1} a_{i, s} A_{s, k}
$$

and for $i=n-p+1, \cdots, n$ :

$$
A_{p+i, k}=\sum_{s=1}^{n} a_{i, s} A_{s, k} .
$$

Here $\delta_{i, k}$ is the Kronecker symbol and we put $a_{i, j}=0$ if $i-j>q$.

The relations above can be written in the matrix form

$$
\boldsymbol{B} A_{k}=\left(\begin{array}{c}
0 \\
\vdots \\
0 \\
A_{n+1, k} \\
A_{n+2, k} \\
\vdots \\
A_{n+p, k}
\end{array}\right),
$$

where

$$
A_{k}=\left[A_{1, k}, A_{2, k}, \cdots, A_{n, k}\right]^{\mathrm{T}} .
$$

We shall note $\boldsymbol{Q}$ the $p \times p$ matrix $\left(A_{n+i, j}\right)_{1 \leq i, j \leq p}$.

The determinant of the matrix $\boldsymbol{Q}$ is related to the determinant of the matrix $\boldsymbol{B}$. This is the purpose of the next section.

\section{Remark}

Suppose that we have $a_{i, p+i}=0$ for some $i=1, \cdots, n-p$, Let $\boldsymbol{B}(\varepsilon)$ be the matrix obtained from $\boldsymbol{B}$ by replacing $a_{i, p+i}$ such that $a_{i, p+i}=\varepsilon$ with $\varepsilon \succ 0$. We have $\lim _{\varepsilon \rightarrow 0} \boldsymbol{B}(\varepsilon)=\boldsymbol{B}$.

Then, we can compute $\boldsymbol{B}^{-1}$ tending $\varepsilon$ e to 0 in $\boldsymbol{B}(\varepsilon)$.

\section{Determinant of a Banded Matrix}

Theorem. Let the banded matrix $\boldsymbol{B}$ and the associated sequence $\left(A_{i, k}\right)_{1 \leq i \leq n+p, 1 \leq k \leq p}$. Then

$$
\operatorname{det}(\boldsymbol{B})=(-1)^{p(n-p)}\left(\prod_{i=1}^{n-p} a_{i, i+p}\right) \operatorname{det}(\boldsymbol{Q}) .
$$

Proof. Let $\boldsymbol{T}$ the $n \times n$ triangular matrix

$$
\boldsymbol{T}=\left(\begin{array}{cc}
\boldsymbol{I}_{p} & \mathbf{0} \\
\boldsymbol{D} & \boldsymbol{I}_{n-p}
\end{array}\right)
$$


where $\boldsymbol{I}_{r}$ denotes the $r \times r$ identity matrix and $\boldsymbol{D}$ is the $(n-p) \times p$ matrix

$$
\left(\begin{array}{cccc}
A_{p+1,1} & A_{p+1,2} & \ldots & A_{p+1, p} \\
A_{p+2,1} & A_{p+2,2} & \ldots & A_{p+2, p} \\
\vdots & \vdots & \vdots & \vdots \\
A_{n, 1} & A_{n, 2} & \cdots & A_{n, p}
\end{array}\right) .
$$

Report that

$$
\left(\begin{array}{cccc}
A_{1,1} & A_{1,2} & \ldots & A_{1, p} \\
A_{2,1} & A_{2,2} & \ldots & A_{2, p} \\
\vdots & \vdots & \vdots & \vdots \\
A_{p, 1} & A_{p, 2} & \cdots & A_{p, p}
\end{array}\right)=\boldsymbol{I}_{p},
$$

and thus using the relation Equation 1 we get

$$
B T=\left(\begin{array}{ll}
0 & C \\
Q & R
\end{array}\right)
$$

where $C$ is the $(n-p) \times(n-p)$ triangular submatrix

$$
\left(\begin{array}{cccc}
a_{1, p+1} & 0 & \cdots & 0 \\
a_{2, p+1} & a_{2, p+2} & \cdots & \vdots \\
\vdots & \vdots & \ddots & 0 \\
a_{n-p, p+1} & a_{n-p, p+2} & \cdots & a_{n-p, n}
\end{array}\right)
$$

and $\boldsymbol{R}$ is a $p \times(n-p)$ matrix.

Obviously, $\operatorname{det}(\boldsymbol{T})=1$ and $\operatorname{det}(\boldsymbol{B} \boldsymbol{T})=(-1)^{p(n-p)} \operatorname{det}(\boldsymbol{C}) \operatorname{det}(\boldsymbol{Q})$. The result follows.

Example. For $p=q=2$, formula of the determinant of the pentadiagonal matrix is presented in [6].

\section{Inverse of a Banded Matrix}

Assume that the matrix $\boldsymbol{B}$ is invertible and let us denote by $C_{j}$ the $j$-th column vector of the inverse matrix $\boldsymbol{B}^{-1}$.

From the relation $\boldsymbol{B}^{-1} \boldsymbol{B}=\boldsymbol{I}_{n}$, where $\boldsymbol{I}_{n}$ denotes the identity matrix of order $n$, we get the relations:

$$
C_{n-j-p}=\frac{1}{a_{n-j-p, n-j}}\left(E_{n-j}-\sum_{i=n-j-p+1}^{n} a_{i, n-j} C_{i}\right) \text {, }
$$

for $j=0, \cdots, n-p-1$, where $E_{j}=\left[\left(\delta_{i, j}\right)_{1 \leq i \leq n}\right]^{\mathrm{T}} \in \mathbb{C}^{n}$ is the vector of order $j$ of the canonical basis of $\mathbb{C}^{n}$.

It follow from $\mathrm{r} 1$ that knowing the $p$ last columns $C_{n}, C_{n-1}, \cdots, C_{n-p+1}$ determine recursively all other columns $C_{n-p}, C_{n-p-1}, \cdots, C_{1}$. We give a straightforward recurrence formulae for computing $C_{n}, C_{n-1}, \cdots, C_{n-p+1}$.

Since the matrix $\boldsymbol{B}$ is invertible, we obtain from the previous section that the matrix $\boldsymbol{Q}$ is invertible too. We shall denotes $X_{j}=\left[x_{1, j}, \cdots, x_{p, j}\right]^{\mathrm{T}}$ the $j$-th column vector of the matrix $\mathbf{Q}^{-1}$.

Theorem. The $j$-th vector column of the inverse matrix $\boldsymbol{B}^{-1}, 1 \leq j \leq p$, is given by

$$
C_{n-p+j}=\sum_{k=1}^{p} X_{k, j} A_{k}
$$

Proof. We get from the relation Equation (1): 


$$
\boldsymbol{B} \cdot \sum_{k=1}^{p} X_{k, j} \boldsymbol{A}_{k}=\left(\begin{array}{c}
0 \\
\vdots \\
0 \\
\sum_{k=1}^{p} x_{k, j} A_{n+1, k} \\
\sum_{k=1}^{p} x_{k, j} A_{n+2, k} \\
\vdots \\
\sum_{k=1}^{p} x_{k, j} A_{n+p, k}
\end{array}\right) .
$$

The result follows from the fact that

$$
\left(\begin{array}{c}
\sum_{k=1}^{p} x_{k, j} A_{n+1, k} \\
\sum_{k=1}^{p} x_{k, j} A_{n+2, k} \\
\vdots \\
\sum_{k=1}^{p} x_{k, j} A_{n+p, k}
\end{array}\right)=\boldsymbol{Q}\left(\begin{array}{c}
x_{1, j} \\
x_{2, j} \\
\vdots \\
x_{p, j}
\end{array}\right)=\left[\left(\delta_{i, j}\right)_{1 \leq i \leq p}\right]^{\mathrm{T}} .
$$

We will use the relations Equation (2) and r1 to explicit the coefficients $c_{s, j}$ for the inverse $\boldsymbol{B}^{-1}$. we have for $1 \leq j \leq p$ and $1 \leq s \leq n$ :

$$
C_{s, n-p+j}=\sum_{k=1}^{p} X_{k, j} A_{s, k}
$$

and for $j=0, \cdots, n-p-1$ :

$$
C_{s, n-j-p}=\frac{1}{a_{n-j-p, n-j}}\left(\delta_{s, n-j}-\sum_{i=n-j-p+1}^{n} a_{i, n-j} c_{s, i}\right) .
$$

Here are the different steps of our algorithm. The implementation is left to the readers' choice.

- Compute $A_{i, k}$ by the relations (1), (2) and (3). If $\operatorname{det}\left(\left(A_{n+i, j}\right)_{1 \leq i, j \leq p}\right)=0$ then $\boldsymbol{B}$ is non invertible.

- Compute the inverse $\left(x_{i, j}\right)_{1 \leq i, j \leq p}$ of the matrix $\left(A_{n+i, j}\right)_{1 \leq i, j \leq p}$.

- Compute $c_{s, n+j-p}$ for $1 \leq j \leq p$ and $1 \leq s \leq n$ by the relation (4).

- Compute $c_{s, n-j-p}$ for $j=0, \cdots, n-p-1$ and $1 \leq s \leq n$ by the relation (5).

\section{Conclusion}

If we fix the bandwidth $p+q$, one can show easily that the complexity of the algorithm is $O\left(n^{2}\right)$. Of course, other algorithms of similar complexity exist (methods based on $L U$ decomposition for example). However, the new method provides more benefits to others: First, the recursive formulas are simple and can be implemented effectively in a parallel machine to reduce the cost. Also, we can solve these relations for some matrices to get the explicit determinant of those matrices.

\section{References}

[1] Aiat Hadj, D. and Elouafi, M. (2008) A Fast Numerical Algorithm for the Inverse of a Tridiagonal and Pentadiagonal Matrix. Applied Mathematics and Computation, 202, 441-445. http://dx.doi.org/10.1016/j.amc.2008.02.026 
[2] Gravvanis, G.A. (2003) On the Solution of Boundary Value Problems by Using Fast Generalized Approximate Inverse Banded Matrix Techniques. The Journal of Supercomputing, 25, 119-129. http://dx.doi.org/10.1023/A:1023936410006

[3] Ran, R.S. and Huang, T.Z. (2009) An Inversion Algorithm for a Banded Matrix. Computers and Mathematics with Applications, 58, 1699-1710. http://dx.doi.org/10.1016/j.camwa.2009.07.069

[4] Trench, W.F. (1974) Inversion of Toeplitz Band Matrices. Mathematics of Computation, 28, 1089-1095. http://dx.doi.org/10.1090/S0025-5718-1974-0347066-8

[5] Kratz, W. (2001) Banded Matrices and Difference Equations. Linear Algebra and its Applications, 337, 1-20. http://dx.doi.org/10.1016/s0024-3795(01)00328-7

[6] Aiat Hadj, A.D. and Elouafi, M. (2008) On the Characteristic Polynomial, Eigenvectors and Determinant of a Pentadiagonal Matrix. Applied Mathematics and Computation, 198, 634-642. http://dx.doi.org/10.1016/j.amc.2007.09.005 